\title{
Eficacia de un programa de intervención para mujeres maltratadas por su pareja
}

\section{Efficacy of an intervention program for battered women}

\author{
M. Pilar Matud \\ Vanesa Padilla \\ Lorena Medina \\ Demelza Fortes \\ Departamento de Personalidad, Evaluación y Tratamientos Psicológicos. \\ Universidad de La Laguna, España
}

Rec. (04 de septiembre de 2013) Acept. (15 de noviembre de 2016)

\begin{abstract}
Resumen
Estudio en el que se analiza la eficacia de un tratamiento psicológico para mujeres maltratadas por su pareja. La muestra está formada por 107 mujeres maltratadas por su pareja con edades comprendidas entre 23 y 64 años (media $=39.64, D T=9.87)$ y características sociodemográficas diversas. Se utilizó un diseño cuasiexperimental con dos grupos independientes (intervención y control) y medidas repetidas (pretratamiento, posttratamiento y seguimientos a los 3 y 6 meses de finalizada la intervención). Las mujeres del grupo con intervención mostraron disminuciones significativas, tanto desde el punto de vista estadístico como clínico, en la sintomatología de estrés postraumático de reexperimentación, evitación y aumento de la activación. También disminuyó su sintomatología depresiva y de ansiedad y aumentó su autoestima y apoyo social. Y dicha mejora se mantuvo en los seguimientos realizados a los 3 y 6 meses de finalizado el programa de intervención.

Palabras clave: mujeres maltratadas, violencia de género, intervención psicológica.
\end{abstract}

\begin{abstract}
This study analyzed the efficacy of a psychological treatment program for battered women. The sample consisted of 107 battered women aged between 23 and 64 years-old (mean age $=39.64, S D=9.87$ ) and with diverse sociodemographic characteristics. A quasi-experimental design of two independent groups (intervention and control) and with repeated measures (pre-treatment, post-treatment and three and six month follow-up) has been used. Women in the intervention group experienced statistically and clinical significant decreases in posttraumatic stress symptoms of reexperienced the traumatic event, avoidance and symptoms of increased arousal. Also experienced significant and clinical decreases in depressive and anxiety symptoms and significant increases in self-esteem and social support. Gains were maintained at 3- and 6-months follow-ups.

Key words: battered women, gender violence, psychological intervention.
\end{abstract}

* Correspondencia: M. Pilar Matud, Facultad de Psicología, Universidad de La Laguna, Campus de Guajara, 38205 La Laguna, Tenerife, España. e-mail: pmatud@ull.es, Teléfono: 34922317487, Fax: 34922317461 


\section{Introducción}

La violencia de género es un grave problema en la sociedad actual y una de las formas más comunes es el maltrato hacia la mujer, por su pareja. Walker (1994) define dicha violencia como el abuso físico, sexual y/o psicológico por el hombre con el que se tiene una relación íntima; y afirma que la meta de tales abusos es ejercer poder y control sobre la víctima.

El maltrato por su pareja afecta a mujeres de todas las culturas, etnias y características sociodemográficas, si bien no todas las mujeres que son maltratadas por su pareja se reconocen como tal (e. g., Instituto de la Mujer, 2006; Mathias, Bedone, Osis y Fernandes, 2013). En un estudio realizado en diez países por la Organización Mundial de la Salud (OMS, 2005), se encontró que la proporción de mujeres que habían sufrido en algún momento de sus vidas violencia física, sexual, o ambas por parte de su pareja, oscilaba entre el $15 \%$ y el $71 \%$, correspondiendo las tasas más bajas a Japón y las más altas a Etiopía, Perú y Tanzania. También en España es un problema importante, si bien aún se desconoce con exactitud su prevalencia. En un estudio con usuarias de Atención Primaria se encontró que entre el $10.7 \%$ y el $28.6 \%$ de las mujeres informaron haber sufrido violencia de un compañero íntimo durante el último año y del $18 \%$ al $40.2 \%$ alguna vez en la vida, tasas que son menores cuando los estudios se realizan en la población general. Así, en la encuesta sobre Violencia contra las Mujeres que realizó de forma periódica el Instituto de la Mujer, se encontró que el porcentaje de mujeres maltratadas fue del $12.4 \%$ en 1999 , del $11.1 \%$ en 2002 y del $9.6 \%$ en 2006 siendo el agresor, en la mayor parte de los casos, la pareja o ex pareja (Instituto de la Mujer, 2006).

Existe evidencia empírica de que el maltrato a la mujer por su pareja incide de forma importante en la salud de las mujeres. Además de las posibles lesiones que pueden causar lesiones directas, les afecta de forma indirecta a través del estrés generado (Sutherland, Bybee y Sullivan, 2002), y es también un factor de riesgo a largo plazo (Campbell et al., 2002). Además de existir evidencia de peor salud física en las mujeres maltratadas por su pareja respecto a las que no han sufrido dicha violencia (Gerber, Wittenberg, Ganz, Williams y McCloskey, 2007), también se han detectado problemas de salud mental. Los trastornos citados con mayor frecuencia son trastorno por estrés postraumático (TEP) y depresión (Golding, 1999). Además, se ha encontrado sintomatología depresiva, ansiosa y baja autoestima (Bermúdez, Matud y Buela-Casal, 2009 ; Dutton, 1992; Ludemir, Schraiber, D’Oliveira, Franca-Junior y Jansen, 2008; Matud, 2004a). También se ha constatado abuso de sustancias (El-Bassel,
Gilbert, Wu, Go y Hill, 2005; Golding, 1999) e intentos de suicidio (Ellsberg, Jansen, Heise, Watts y García-Moreno, 2008; Golding, 1999), y se ha planteado que muchas mujeres maltratadas por su pareja tienen sentimientos de culpa, aislamiento social y dependencia emocional del maltratador (Echeburúa y Corral, 1998).

Aunque son diversos los programas de intervención implementados en la violencia de pareja (cfrs., Bermúdez et al., 2013; Cohen, Field, Campbell y Hien, 2013; Ibaceta, 2011), son muchas las cuestiones por responder en la intervención psicológica con mujeres maltratadas por su pareja. Lundy y Grossman (2001), en un estudio de revisión, encontraron que aunque se habían utilizado más de 16 modelos había muy poca investigación clínica sobre su eficacia. Aunque la situación parece haber mejorado durante la última década, habiéndose publicado varios estudios sobre la eficacia de las intervenciones psicológicas, generalmente éstas tienen limitaciones y/o requisitos en su aplicación. Algunos son para mujeres maltratadas por su pareja que tienen TEP (Cohen et al., 2013; Kubany, Hill y Owens, 2003; Kubany et al., 2004; Labrador, Fernández-Velasco y Rincón, 2006); Johnson y Zlotnick, 2006). Pero, aunque han mostrado su eficacia, no todas las mujeres maltratadas por su pareja tienen TEP. Además, su rango de aplicación se restringe ya que algunas intervenciones requieren cumplir requisitos tales como estar en casas de acogida (Johnson y Zlotnick, 2006), o haber dejado previamente la relación con el agresor (Kubany et al., 2003; Kubany et al., 2004); o se trata de estudios piloto (Guerrero y García, 2008; Zlotnick, Capezza y Parker, 2011). También se ha criticado que algunos programas incorporan, al igual que los tratamientos tradicionales del TEP, exposición, que se considera contraindicada en las mujeres maltratadas por su pareja ya que la habituación a los estímulos temidos puede aumentar el riesgo de victimización posterior (Johnson y Zlotnick, 2006). Además, tal y como plantea Haj-Yahia (2011), la mayoría de dichos programas, así como los principios y filosofía que los sustentan, se han desarrollado y validado con mujeres de culturas occidentales individualistas por lo que se desconoce la medida en que pueden ser aplicables a mujeres de sociedades más colectivistas.

Nuestro grupo de investigación (Matud, 2004b), tras el estudio empírico del maltrato a la mujer por su pareja y el impacto en la salud de sus víctimas, ha diseñado un programa de intervención psicológica en el que, aunque predomina la aplicación de técnicas cognitivo-conductuales, se incorporan los principios y estrategias generadas desde la psicología positiva (véase Park y Sun, 2013; Tarragona, 2013) tales como; promover sus habilidades, fortalezas y 
recursos y enfatizar las construcción de soluciones y la agencia personal. Dicha intervención puede ser aplicada a mujeres maltratadas por su pareja en diversidad de circunstancias y de forma individual y grupal, o mediante la combinación de ambos formatos, y ha sido publicado como Guía de tratamiento grupal (Matud Padilla y Gutiérrez, 2005). Siguiendo las propuestas de autoras como Dutton (1992) y Walker (1994), dicho programa tiene tres objetivos principales: 1) ayudarles a aumentar su seguridad, 2) ayudarles a recuperar el control de su vida, y 3 ) remediar el impacto psicológico del abuso. Aunque los resultados de la evaluación inicial de los efectos de dicho programa (Matud, 2004b) evidenciaron su eficacia en la reducción de la sintomatología y en el aumento de la autoestima y el apoyo social, dicho estudio se realizó con una muestra muy reducida de mujeres por lo que es importante realizar una nueva valoración de su eficacia. Por tanto, el objetivo de la presente investigación cuasi-experimental es valorar la eficacia de la intervención psicológica con mujeres maltratadas por su pareja siguiendo la Guía de tratamiento de Matud et al. (2005).

\section{Método}

\section{Participantes}

La muestra está formada por 107 mujeres maltratadas por su pareja, usuarias de servicios públicos para víctimas de violencia de género de la Comunidad Canaria. Sus edades oscilaban entre 23 y 64 años (edad media $=39.64$ años, $D T=9.87)$. Su nivel de estudios era diverso, si bien, lo más común, era que únicamente tuviesen estudios básicos. También había diversidad en su estado civil y profesión, aunque solo el $46 \%$ tenía empleo. Lo más frecuente es que las mujeres hubiesen sufrido maltrato físico, psicológico y sexual, lo que se daba en el 43.8\%; el 32.4\% informó de haber sufrido maltrato físico y psicológico; únicamente maltrato psicológico el $18.1 \%$; y maltrato psicológico y sexual el 5.7\%.

De las 107 mujeres, 72 participaron en alguno de los grupos en que se implementó la guía de tratamiento de Matud et al. (2005) y forman el grupo de intervención protocolizado. El resto son mujeres que, o bien acudieron a algún centro donde no estaba disponible dicho programa (n =17), o accedieron a algún centro donde, tras la evaluación psicológica o tras la primera o segunda sesión de intervención grupal, abandonaron el programa $(\mathrm{n}=18)$. Estas 35 mujeres conforman el grupo de control denominado "tratamiento no protocolizado".

Los criterios de inclusión en el grupo de intervención protocolizado fueron: 1) haber sido o estar siendo víctima de malos tratos por parte de su pareja; 2) ser mayor de edad; 3) acceder participar en un programa de intervención grupal.

\section{Instrumentos}

Para conocer las características sociodemográficas y el maltrato sufrido, así como la salud mental de las participantes, se utilizaron instrumentos comúnmente utilizados y cuya fiabilidad y validez ha sido probada o que habían sido validados previamente con mujeres maltratadas por su pareja (Matud, 1999; 2004b).

Entrevista semiestructurada de historia y tipo de maltrato (Matud, 1999). Recoge los aspectos fundamentales del maltrato de la pareja, tanto actual como su historia y dinámica funcional.

Escala de gravedad de sintomas del trastorno de estrés postraumático (Echeburúa, Corral, Amor, Zubizarreta y Sarasúa, 1997). Escala de evaluación hetereoaplicada que consta de 17 ítems basados en los criterios diagnósticos del DSM-IV (APA, 1994).

Inventario para la ansiedad de Beck (BAI; Beck y Steer, 1990). Está formado por 21 ítems que evalúan sintomatología ansiosa y su gravedad. Se ha utilizado la versión española de Sanz y Navarro (2003) que ha sido validada para la población adulta española (Magán, Sanz y García-Vera, 2008), siendo su consistencia interna de .93.

Inventario para la depresión de Beck-Segunda Edición (BDI-II; Beck, Steer y Brown, 1996). Instrumento de autoinforme de 21 ítems diseñado para evaluar la gravedad de la sintomatología depresiva. La consistencia interna de la versión española utilizada es de .87 (Sanz, Perdigón y Vázquez, 2003).

Inventario de autoestima (SEQ-MR). Versión reducida y validada con mujeres maltratadas del Self-esteem Inventory de Rector y Roger (1993). Consta de 25 ítems que se agrupan en un factor de segundo orden cuya consistencia interna es de .93 .

Inventario de Apoyo Social (Matud, 1999). Consta de 12 elementos que evalúan la percepción de apoyo en diversas áreas y se agrupan en un factor de segundo orden cuya consistencia interna es de .90 .

Inventario de evaluación del maltrato a la mujer por su pareja (APCM, Matud, 2004b). Consta de 57 ítems 15 de los cuales se refieren a maltrato físico y cuya consistencia interna es de .94; 38 de maltrato psicológico con una 
consistencia interna de .93 ; y 4 de violencia sexual, cuya consistencia interna es de .87 .

\section{Diseño}

El diseño es cuasiexperimental de dos grupos independientes (grupo experimental de intervención psicológica grupal protocolizada y grupo control sin intervención protocolizada) con medidas repetidas (pretratamiento, postratamiento y seguimientos a los tres y seis meses de finalizar el programa de intervención), si bien únicamente en las evaluaciones pretratamiento y postratamiento hay una condición de no tratamiento.

\section{Procedimiento}

Se contactó con las participantes a través de cuatro centros públicos para víctimas de violencia de género de la Comunidad Canaria con los que la Universidad de La Laguna tenía firmados convenios de investigación. Las mujeres que solicitaron asistencia psicológica fueron derivadas a la psicóloga del centro, quien les explicó el programa de intervención. Tras firmar el consentimiento informado, se las citó de forma individual para realizar la evaluación psicológica inicial.

\section{Programa de tratamiento}

El programa de intervención psicológica se realizó siguiendo la guía de tratamiento de Matud et al. (2005) en el propio centro donde fueron evaluadas. Aunque dicha guía está estructurada en de 10 sesiones, el número llevado a cabo en cada grupo fue sensiblemente mayor, oscilando entre 15 y 20 ya que cada sesión se adaptaba al ritmo de las participantes. El programa consta de los siguientes componentes: 1) Psicoeducación en género y en violencia de género. Para aumentar su seguridad y toma de conciencia de la violencia de género se explica qué es la violencia de género y los malos tratos de la pareja analizando y debatiendo sus características principales y las consecuencias en sus víctimas, así como las medidas de seguridad, aspecto que también es tratado de forma individual en el caso de que se detecte la presencia de riesgo para la seguridad de alguna de las mujeres. Para modificar las creencias tradicionales acerca de los roles de género y las actitudes sexistas, se analiza cómo la socialización tradicional y los estereotipos de género, además de suponer una limitación para el desarrollo pleno de los seres humanos, genera y mantiene la violencia contra las mujeres. 2) Estrategias y técnicas terapéuticas cuyo fin es reducir y/o eliminar sus síntomas. Entre las técnicas incluidas están la reestructuración cognitiva y el entrenamiento en solución de problemas, además de diversas técnicas de autocontrol y de disminución del nivel de activación tales como entrenamiento en respiración y diversas formas de relajación. 3) Estrategias y técnicas para aumentar su autoestima y seguridad en sí mismas tales como fomento de un autoconcepto adecuado, autorefuerzo y autocuidado y programación de actividades. 4) Fomentar una comunicación y habilidades sociales adecuadas. 5) Entrenamiento en solución de problemas. Además se incluye psicoeducación para fomentar la independencia emocional y técnicas de prevención de recaídas.

La intervención grupal fue llevada a cabo en pequeño grupo conducido por dos psicólogas formadas en violencia de género y en la intervención a ser implementada. $\mathrm{Su}$ periodicidad fue semanal y su duración aproximada de dos horas. Hubo un total de 16 grupos que fueron conducidos por 9 psicólogas.

Inmediatamente después de finalizar el grupo de intervención, las mujeres fueron evaluadas de nuevo así como a los tres y seis meses de haber concluido dicho programa. En dichas evaluaciones no fue posible localizar a algunas de las mujeres, generalmente por haber cambiado de domicilio. Las mujeres que no participaron en los grupos de intervención psicológica protocolizada fueron evaluadas en una segunda ocasión, en un intervalo temporal de entre 4 y 14 meses tras la evaluación inicial.

Se aseguró el anonimato y confidencialidad de las participantes por medio de un sistema de códigos que permitía que la identificación de las participantes únicamente fuese posible en el centro donde se realizó la evaluación y la intervención, pero no en la Universidad de La Laguna, centro donde fueron analizados los datos.

\section{Resultados}

\section{Análisis de la homogeneidad de los grupos}

Para conocer la homogeneidad inicial entre los grupos con intervención psicológica protocolizada y los que no participaron en dicha intervención se compararon los datos obtenidos en la evaluación inicial. En la Tabla 1 se muestran los datos de las características sociodemográficas y del maltrato. Como puede observarse, únicamente se dieron diferencias estadísticamente significativas en los años de maltrato, que era mayor en el grupo de intervención 
protocolizada. Tampoco se encontraron diferencias entre ambos grupos en el nivel de estudios (básicos frente a medios o universitarios), $\chi^{2}(1, N=106)=4.17, p>.05$; en convivir o no con la pareja violenta, $\chi^{2}(1, N=107)=1.44$, $p>.05$; ni en haberlo denunciado o no $\chi^{2}(1, N=103)=$ $0.59, p>.05$.

Al comparar ambos grupos en sintomatología, autoestima y apoyo social, únicamente se encontraron diferencias estadísticamente significativas en ansiedad y en apoyo social, que era mayor en las mujeres del grupo de intervención protocolizado.

\section{Resultados de la intervención}

Para analizar los cambios obtenidos en las variables consideradas como dependientes entre antes e inmediatamente después del programa de intervención psicológica en las mujeres que participaron en el grupo de intervención protocolizada, y entre el primer y segundo pase de pruebas en el caso de las mujeres que no participaron en dichos grupos, se hicieron análisis de varianza de medidas repetidas con una variable independiente intragrupo con dos niveles (pretratamiento y postratamiento) y una variable independiente inter-grupo con dos niveles (intervención protocolizada e intervención no protocolizada).

Cuando las variables dependientes fueron las puntuaciones en estrés postraumático, se encontraron interacciones estadísticamente significativas entre el tipo de grupo y el momento de evaluación, tanto en la puntuación global de la Escala de gravedad de síntomas del TEP, $F(1,93)=30.92$, $p<.001$; como en las subescalas de reexperimentación, $F(1,96)=24.04, p<.001$; evitación, $F(1,93)=16.50, p<$ .001 ; y aumento de la activación, $F(1,93)=19.35, p<.001$.

El MANOVA para el análisis de los efectos simples, mostró la existencia de diferencias estadísticamente significativas entre el pretratamiento y el postratamiento en el grupo de mujeres con intervención protocolizada, $F(1,93)$ $=154.92, p<.001$, pero no en el grupo control de mujeres sin intervención protocolizada, $F(1,93)=2.49, p>.05$. Los ANOVA mostraron que las mujeres maltratadas por su pareja, que participaron en algún grupo en el que se ha llevado a cabo la intervención grupal protocolizada, presentaron inmediatamente, tras ésta, una disminución estadísticamente significativa de la sintomatología de reexperimentación, $F(1,96)=104.57, p<.001$; evitación $F(1,96)=104.57, p<$ .001 ; y activación, $F(1,96)=104.57, p<.001$ (véase Tabla 2).

Cuando se consideró como variable dependiente la puntuación en depresión, también se encontró que la interacción entre el tipo de grupo y el momento de evaluación era estadísticamente significativa, $F(1,87)=48.30, p<.001$. El ANOVA para el análisis de los efectos simples mostró que en el grupo de mujeres sin intervención protocolizada las diferencias no eran estadísticamente significativas, $F(1,87)$ $=0.77, p>.05$, aunque sí lo eran entre el pretratamiento y el postratamiento en el grupo de las mujeres con intervención protocolizada, $F(1,87)=169.94, p<.001$. Como se observa

Tabla 1. Medias (desviaciones típicas) y comparaciones del grupo de mujeres con intervención grupal protocolizada $(n=72)$ $y$ sin intervención protocolizada $(n=35)$ en la evaluación inicial.

\begin{tabular}{|c|c|c|c|}
\hline & $\begin{array}{c}\text { Con intervención } \\
\text { protocolizada }\end{array}$ & $\begin{array}{c}\text { Sin intervención } \\
\text { protocolizada }\end{array}$ & $F$ \\
\hline Edad & $40.14(9.51)$ & $38.91(10.68)$ & 0.35 \\
\hline Número de hijos/as & $1.91(1.24)$ & $1.76(1.44)$ & 0.29 \\
\hline Maltrato psicológico & $98.16(29.52)$ & $88.44(31.56)$ & 2.27 \\
\hline Maltrato físico & $11.31(10.22)$ & $12.12(12.84)$ & 0.11 \\
\hline Maltrato sexual & $3.78(4.14)$ & $3.41(3.80)$ & 0.15 \\
\hline Años de maltrato & $15.16(10.82)$ & $9.32(10.18)$ & $6.28^{*}$ \\
\hline $\begin{array}{l}\mathrm{N}^{0} \text { de meses desde el último } \\
\text { episodio de maltrato }\end{array}$ & $9.81(26.56)$ & $9.94(23.81)$ & 0.01 \\
\hline
\end{tabular}

Nota. $* \mathrm{p}<.05$ 
en la Tabla 2, la puntuación en el BDI se redujo de forma importante tras el programa grupal de intervención.

Al considerar como variable dependiente la puntuación en ansiedad, también se encontró interacción estadísticamente significativa entre el tipo de grupo y el momento de evaluación, $F(1,83)=54.01, p<.001$. El ANOVA para el análisis de los efectos simples mostró la existencia de diferencias, estadísticamente significativas, entre el pretratamiento y el postratamiento en el grupo con intervención protocolizada, $F(1,83)=189.73, p<.001$, mientras que en el grupo sin intervención protocolizada las diferencias no eran estadísticamente significativas, $F(1,83)=1.77, p$ $>$.05. Como puede observarse en la Tabla 2, las mujeres del grupo con intervención protocolizada tenían, tras ésta, menor ansiedad.

El ANOVA en que la variable dependiente fue la puntuación en el cuestionario de autoestima, también mostró interacción estadísticamente significativa entre el tipo de grupo y el momento de evaluación, $F(1,89)=33.64 p<.001$. El ANOVA para el análisis de los efectos simples mostró la existencia de diferencias estadísticamente significativas entre el pretratamiento y el postratamiento en las mujeres con intervención protocolizada, $F(1,89)=110.19, p<.001$, mientras que en las que no tuvieron dicha intervención, las diferencias no fueron estadísticamente significativas. $F(1,89)=0.75, p>.05$. Las mujeres maltratadas por su pareja que participaron en algún grupo en el que se llevó a cabo la intervención psicológica grupal siguiendo el manual de tratamiento, presentaban inmediatamente, tras ésta, un aumento de su autoestima (véase Tabla 2).

Al considerar como variable dependiente el apoyo social no se encontraron interacciones estadísticamente significativas entre el tipo de grupo y el momento de evaluación, $F(1,98)$ $=0.93 p>.05$. Pero sí fueron estadísticamente significativos los efectos principales del momento de evaluación, $F(1,98)$ $=17.25, p<.001$, y del tipo de grupo, $F(1,98)=25.73, p<$ .001 . Como puede observarse en la tabla 2 , aunque ambos grupos de mujeres informan de mayor percepción de apoyo social en la segunda evaluación respecto a la primera, dicho aumento es mayor en el grupo de mujeres que participaron en el grupo de intervención protocolizada.

\section{Significación clínica}

En la evaluación inicial el $47.8 \%$ de las mujeres que participaron en los grupos de intervención protocolizada y el $53.3 \%$ de las que no participaron en dichos grupos cumplían con los criterios diagnósticos de TEP. Inmediatamente tras el programa de intervención psicológica, el 14.3\% de las mujeres que participaron en dicha intervención el 38.2\% de las que no tuvieron intervención psicológica protocolizada, el cumplían con los criterios diagnósticos de TEP.

La clasificación de la gravedad de la sintomatología depresiva evaluada a través del BDI mostró que el 71.4\% de las mujeres que participaron en el grupo de intervención protocolizada tenía depresión moderada o grave en la evaluación inicial, porcentaje que fue del $8.4 \%$ tras dicha intervención. Los porcentajes de ansiedad moderada o grave, según el BAI, fueron del $81.1 \%$ antes de la intervención y del $14.1 \%$ tras ésta.

\section{Seguimientos a los 3 y 6 meses}

Para analizar los cambios entre las puntuaciones de la evaluación inicial y los seguimientos realizados a los $3 \mathrm{y}$ 6 meses de finalizado el programa de intervención grupal protocolizada, se realizaron ANOVAs de medidas repetidas entre cada una de las puntuaciones de las variables que se consideraron como dependientes en la evaluación inicial y en cada uno de los dos seguimientos. No fue posible localizar a 9 de las mujeres en el seguimiento realizados a los 3 meses y a 7 , en el realizado a los 6 meses de finalizado el programa de intervención. Además, no se pudieron obtener todos los datos de algunas de las mujeres por lo que el $n$ difiere ligeramente en algunas medidas, como puede observarse en las Tablas 3 y 4.

En la Tabla 3 se presentan las medias, desviaciones típicas y los valores $F$ de las comparaciones de las puntuaciones de antes del tratamiento y del seguimiento a los 3 meses. Como puede observarse, la sintomatología en las tres subescalas del TEPT se ha reducido prácticamente a la mitad, la ansiedad a la tercera parte y la sintomatología depresiva a la cuarta parte de lo detectado en la evaluación inicial. Además, ha aumentado la autoestima y el apoyo social, diferencias que son estadísticamente significativas.

También en los seguimientos realizados a los 6 meses de finalizada la intervención se observó que la sintomatología había disminuido de forma importante y estadísticamente significativa respecto a la evaluación inicial, y la autoestima y el apoyo social había aumentado (véase Tabla 4).

La evaluación de la significación clínica de los cambios en los seguimientos mostró que, en el seguimiento realizado a los 3 meses, el $17.7 \%$ de las mujeres tenía TEP, porcentaje que fue del $10.8 \%$ a los 6 meses de finalizada la intervención. El porcentaje de mujeres con sintomatología depresiva grave o moderada fue del $8 \%$ a los 3 meses y de $6.2 \%$ a los 6 . El 23.8\% de las mujeres tenía ansiedad grave o moderada en el seguimiento realizado a los 3 meses y el 
Tabla 2. Medias (desviaciones tipicas) antes del tratamiento y tras éste en el grupo de mujeres con intervención grupal protocolizada y sin intervención protocolizada.

\begin{tabular}{lcccc}
\hline & \multicolumn{2}{c}{ Con intervención protocolizada } & \multicolumn{2}{c}{ Sin intervención protocolizada } \\
\hline Reexperimentación & Pretratamiento & Postratamiento & Pretratamiento & Postratamiento \\
Evitación & $8.01(3.74)$ & $3.51(3.28)$ & $6.30(3.95)$ & $5.70(4.39)$ \\
Aumento activación & $8.90(4.25)$ & $3.29(3.74)$ & $10.45(4.34)$ & $9.14(5.15)$ \\
Depresión (BDI) & $7.59(3.50)$ & $3.35(2.82)$ & $7.97(2.99)$ & $7.03(3.14)$ \\
Ansiedad (BAI) & $28.36(13.79)$ & $7.51(7.64)$ & $29.50(14.21)$ & $32.10(15.67)$ \\
Autoestima & $31.88(12.96)$ & $10.25(8.32)$ & $29.29(16.83)$ & $33.47(18.08)$ \\
Apoyo social & $36.13(18.00)$ & $56.10(11.37)$ & $33.71(17.56)$ & $30.71(16.94)$ \\
\hline
\end{tabular}

Tabla 3. Medias, desviaciones típicas y ANOVAs entre las puntuaciones antes del tratamiento y en el seguimiento realizado a los 3 meses tras el tratamiento en el grupo de mujeres con intervención grupal protocolizada

\begin{tabular}{|c|c|c|c|c|c|c|c|}
\hline & \multicolumn{2}{|c|}{ Pretratamiento } & \multicolumn{2}{|c|}{ Seguimiento 3 meses } & \multirow[b]{2}{*}{$n$} & \multirow[b]{2}{*}{$g l$} & \multirow[b]{2}{*}{$F$} \\
\hline & Media & $D T$ & Media & $D T$ & & & \\
\hline Reexperimentación & 8.00 & 3.80 & 3.23 & 3.32 & 60 & 59 & $71.64 * * *$ \\
\hline Evitación & 8.60 & 4.17 & 3.38 & 3.78 & 58 & 57 & $59.04 * * *$ \\
\hline Aumento activación & 7.45 & 3.49 & 3.53 & 3.32 & 58 & 57 & $54.77 * * *$ \\
\hline Depresión (BDI) & 27.49 & 14.16 & 6.79 & 7.51 & 61 & 60 & $115.91 * * *$ \\
\hline Ansiedad (BAI) & 32.33 & 13.05 & 11.37 & 9.99 & 61 & 60 & $121.26^{* * *}$ \\
\hline Autoestima & 36.73 & 18.41 & 58.13 & 9.64 & 62 & 61 & $76.35^{* * *}$ \\
\hline Apoyo social & 23.06 & 9.13 & 27.76 & 7.70 & 62 & 61 & $22.86^{* * *}$ \\
\hline
\end{tabular}

Nota. $* * * \mathrm{p}<0.001$.

Tabla 4. Medias, desviaciones típicas y ANOVAs entre las puntuaciones antes del tratamiento y en el seguimiento realizado a los 6 meses tras el tratamiento en el grupo de mujeres con intervención grupal protocolizada

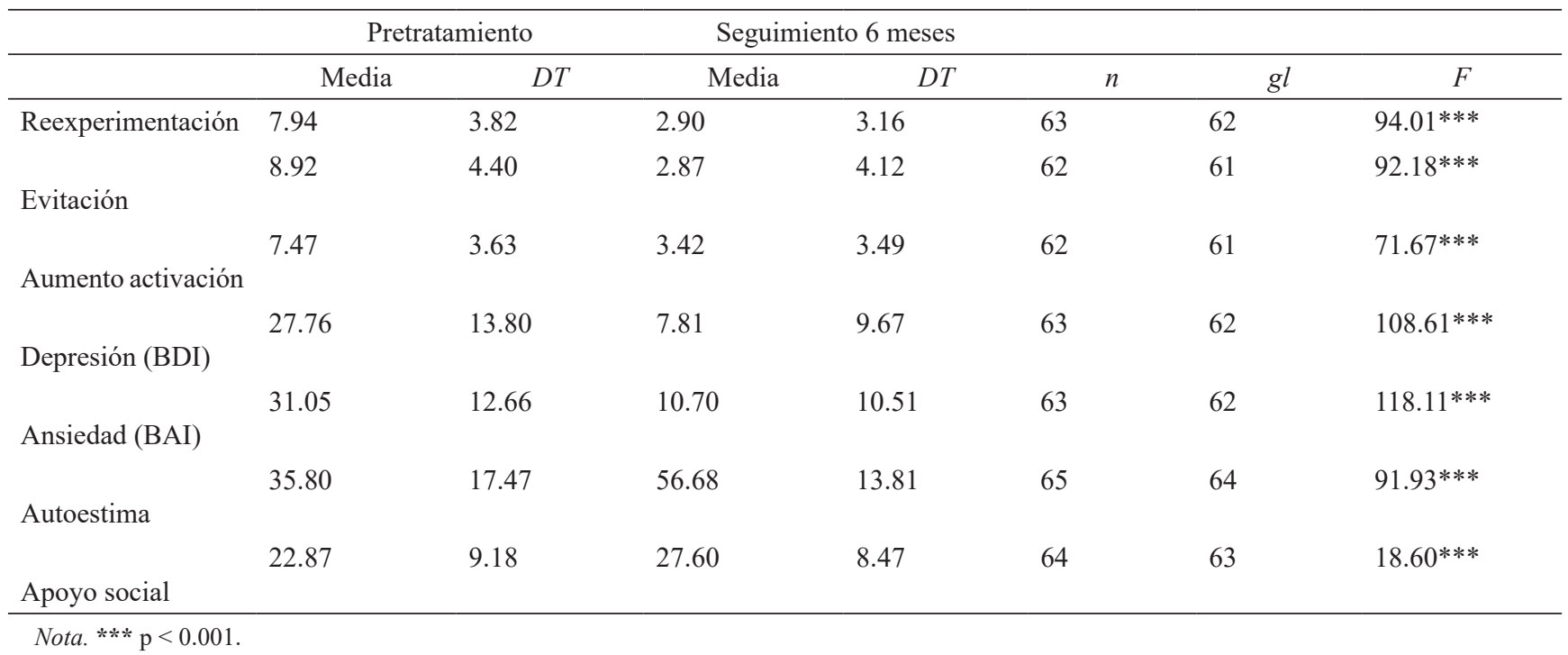


$17 \%$ en el seguimiento realizado a los 6 meses de finalizada la intervención.

Para conocer la satisfacción con el programa de intervención, se pidió a las mujeres que valoraran el tratamiento escribiendo su opinión en una hoja diseñada para ello que se incorporó a la batería de evaluación del postratamiento. El 59.8\% de las mujeres valoró la intervención como muy buena, el 19.4\% como "buena", y el 20.8\% escribió que les hubiese gustado que el programa de intervención tuviese más sesiones.

\section{Discusión}

El objetivo del presente estudio es evaluar empíricamente la eficacia del programa de intervención grupal para mujeres maltratadas por su pareja de Matud et al. (2005). Se trata de una guía de tratamiento psicológico que, centrado en el desarrollo de las potencialidades de la mujer, y priorizando su seguridad, tiene como meta final ayudarle a recuperar el control de su propia vida, además de reducir y/ o eliminar sus síntomas y aumentar su autoestima. Dicha intervención ha mostrado ser efectiva ya que las mujeres que participaron en alguno de los grupos donde se llevó a cabo, mostraron una disminución estadísticamente significativa en la sintomatología de estrés postraumático de reexperimentación, evitación y aumento de la activación, así como de su sintomatología depresiva y de ansiedad. Además, aumentó su autoestima y apoyo social. También se encontró mejoría clínica disminuyendo de forma importante las tasas de estrés postraumático y de mujeres con ansiedad y depresión moderada y grave. Tales cambios se dieron inmediatamente tras la intervención y se mantuvieron en los seguimientos realizados a los tres y seis meses tras su finalización. En esta evaluación el 90\% de las mujeres no tenían TEP, porcentaje que es muy similar al encontrado en otros estudios en que se aborda de forma directa el tratamiento de dicho trastorno (Kubany et al., 2004).

Por el contrario, no se detectó tal mejoría en un grupo de 35 mujeres, usuarias de los mismos servicios, que no participaron en dicha intervención grupal protocolizada y cuyas características sociodemográficas, violencia sufrida y estado de salud era similar, si bien tenían menor sintomatología de ansiedad, apoyo social percibido y habían sufrido la violencia durante menos años. Aunque la mitad de esas mujeres no tuvieron la oportunidad de participar en el grupo de intervención protocolizada, el resto habían dejado tal intervención en la primera o segunda sesión. Aunque se desconoce las razones de dichas diferencias, pudiese suceder que: 1) el no tener suficiente apoyo social les dificultase seguir de forma continuada un programa de intervención con unas fechas y horario definidos; 2) al sentirse menos ansiosas no se implicaron en la intervención protocolizada por no valorar su situación como de suficiente gravedad.

Aunque el presente estudio ha mostrado la eficacia de la guía de tratamiento grupal de Matud et al. (2004 b) para mejorar la salud mental de las mujeres maltratadas por su pareja, tiene una serie de limitaciones: 1) falta de asignación aleatoria a los grupos de tratamiento; procedimiento importante para prevenir la confusión entre los efectos del tratamiento y las diferencias preexistentes (Davis y Auchter, 2010). Una de las dificultades metodológicas en los estudios con muestras clínicas es utilizar un grupo control, ya que conlleva privar de ayuda psicológica a quienes lo precisan. Ello es aún más importante en las mujeres maltratadas por su pareja ya que su seguridad está amenazada. Además, las participantes son mujeres que habían acudido en demanda de ayuda a centros públicos de atención a víctimas de violencia de género lo que puede limitar la generabilidad de los resultados. 2) No todas las mujeres finalizaron el programa de intervención, por lo que los datos obtenidos pueden tener algún sesgo. De las 93 mujeres que comenzaron el tratamiento grupal lo finalizaron 72. Los motivos citados con mayor frecuencia para no continuar en dicho programa fueron comenzar a trabajar, con la consecuente incompatibilidad de horarios, cambio de domicilio, dificultades para el cuidado de sus hijos/ as durante el tiempo de la intervención y graves problemas económicos que les impedían costearse el desplazamiento. 3) Falta de seguimiento a más largo plazo. Además, aún quedan algunas cuestiones por resolver siendo, quizá, la más importante conocer las razones por las que para una minoría de mujeres la intervención no fue efectiva, análisis que debería acometerse en futuros trabajos.

Actualmente se reconoce la necesidad urgente de tratamientos psicológicos basados en la evidencia (McHugh y Barlow, 2010), y la necesidad de poner a prueba y validar nuevos procedimientos y técnicas psicológicas (Pago, 2009). Y ello parece ser aún más crítico en las intervenciones con mujeres maltratadas por su pareja, donde se ha reconocido la necesidad urgente de lograr evidencias de lo que es efectivo en su prevención y en la recuperación de las víctimas (Taft y Hegarty, 2010). 


\section{Referencias}

American Psychiatric Association. (1994). Diagnostic and statistical manual of mental disorders (4. ${ }^{\mathrm{a}}$ ed.). Washington, DC: Author.

Beck, A. T., y Steer, R. A (1990). Manual for Beck Anxiety Inventory. San Antonio, TX: The Psychological Corporation.

Beck, A. T., Steer, R. A., y Brown, G. K. (1996). BDI-II. Beck Depression Inventory. Manual. San Antonio, TX: The Psychological Corporation.

Bermúdez, D., Benjamin, M. T., Porter, S. E., Saunders, P. A., Myers, N. A. L., y Dutton, M. A. (2013). A qualitative analysis of begining mindfulness experiences for women with post-traumatic stress disorder and a history of intimate partner violence. Complementary Therapies in Clinical Practice, 19, 104-108.

Bermúdez, M. P., Matud, M. P., y Buela-Casal, G. (2009). Salud mental de las mujeres maltratadas por su pareja en el Salvador. Revista Mexicana de Psicología, 26, 51-59.

Campbell, J., Jones, A. S., Dienneman, J., Kub, J., Schollenberger, J., O'Campo, P., Gielen, A. C., y Wynne, C. (2002). Intimate partner violence and physical health consequences. Archives of Internal Medicine, 162, 1157-1163.

Cohen, L. R., Field, C., Campbell, A. N. C., y Hien, A. D. (2013). Intimate partner violence outcomes in women with PTSD and substance use: A secondary analysis of NIDA clinical trials network "Women and Trauma" multi-site study. Addictive Behaviors, 38, 2325-2332.

Davis, R. C., y Auchter, B. (2010). National Institute of Justice funding of experimental studies of violence against women: a critical look at implementation issues and policy implications. Journal of Experimental Criminology, 6, 377-395.

Dutton, M. A. (1992). Empowering and healing the batted woman: A model of assessment e intervention. Nueva York: Springer.

Echeburúa, E.. y Corral, P. (1998). Manual de violencia familiar. Madrid: Siglo XXI.

Echeburúa, E., Corral, P., Amor, P. J., Zubizarreta, I., y Sarasua, B. (1997). Escala de gravedad de síntomas del trastorno de estrés postraumático: propiedades psicométricas. Análisis y Modificación de Conducta, 23, 503-526.

El-Bassel, N., Gilbert, L., Wu, E., Go, H., y Hill, J. (2005). Relationship between drug abuse and intimate partner violence: A longitudinal study among women receiving Metadhona. American Journal of Public Health, 95, 465-470.

Ellsberg, M., Jansen, H., Heise, L., Watts, C. H., y García-Moreno, C. (2008). Intimate partner violence and women's physical and mental health in the WHO multi-country study on women's health and domestic violence: an observational study. The Lancet, 371, 1165-1172.

Gerber, M. R., Wittenberg, E., Ganz, M. L., Williams, C. M., y McCloskey, L. A. (2007). Intimate partner violence exposure and change in women's physical symptoms over time. Journal of General Internal Medicine 23, 64-69.

Golding, J. M. (1999). Intimate partner violence as a risk factor for mental disorders: a meta-analysis. Journal of Family Violence, 14, 99-132.

Guerrero, R., y García, J. (2008). Ensayo clínico de la eficacia de la terapia constructivista-sistémica en casos de violencia contra las mujeres. Apuntes de Psicología, 26, 269-280.

Haj-Yahia, M. M. (2011). Contextualizing interventions whit battered women in collectivist societies: Issues and controversies. Aggression and Violent Behavior, 16, 311-339.

Ibaceta, F. (2011). Violencia en la pareja: ¿Es posible la terapia conjunta? Terapia Psicológica, 29, 117-125

Instituto de la Mujer (2006). III Macroencuestra sobre la violencia contra las mujeres. Informe de resultados, abril de 2006. Recuperado desde http://www.inmujer.migualdad.es/mujer/mujeres/estud_inves/ violencia\%20final.pdf.

Johnson, D. M., y Zlonick, C. (2006). A cognitive-behavioral treatment for battered women with PTSD in shelters: findings from a pilot study. Journal of Traumatic Stress, 19, 559-564.
Kubany, E. S., Hill, E. E., y Owens, J. A. (2003). Cognitive trauma therapy for battered women with PTSD: preliminary findings. Journal of Traumatic Stress, 16, 81-91.

Kubany, E. S., Owens, J. A., McCaig, M. A., Hill, E. E, Iannce-Spencer, C., y Tremayne, K. J. (2004). Cognitive trauma therapy for battered women with PTSD (CTT-BW). Journal of Consulting and Clinical Psychology, 72, 3-18.

Labrador, F. J., Férnandez-Velasco, M. R. y Rincón, P. (2006). Eficacia de un programa de intervención individual y breve para el trastorno por estrés postraumático en mujeres víctimas de violencia doméstica. International Journal of Clinical and Health Psychology, 6, 527-547.

Ludemir, A. B., Schraiber, L. B., D’Oliveira, A. F., Franca-Junior, I., y Jansen, H. A. (2008). Violence against women by their intimate partner and common mental disorders. Social Science y Medicine, 66, 1008-1018.

Lundy, M., y Grossman, S. (2001). Clinical research and practice with battered women. What we know, what we need to know. Trauma, Violence, $y$ Abuse, 2, 120-141.

Magán, I., Sanz, J., y García-Vera, M. P. (2008). Psychometric properties of a Spanish version of the Beck Anxiety Inventory (BAI) in general population. The Spanish Journal of Psychology, 11, 626-640.

Mathias, A. K., Bedone, A., Osis, M. J., y Fernandes, A. (2013). Perception of initmate partner violence among women seeking care in the primary healthcare network in Sao Paulo State, Brazil. International Journal of Gynecology and Obstetrics, 121, 214-217.

Matud, M. P. (1999). Impacto psicológico del maltrato a la mujer: un análisis empírico. Informe de investigación. Las Palmas de Gran Canaria: Instituto Canario de la Mujer.

Matud, M. P. (2004a). Impacto de la violencia doméstica en la salud de la mujer maltratada. Psicothema, 16, 397-401.

Matud, M. P. (2004b). Diseño y validación de un programa de intervención psicológica con mujeres víctimas de maltrato por parte de su pareja. Informe de investigación. Madrid: Instituto de la Mujer.

Matud, M. P., Padilla, V., y Gutiérrez, A. B. (2005). Mujeres maltratadas por su pareja. Guía de tratamiento psicológico. Madrid: Minerva Ediciones.

McHugh, R. L., y Barlow, D. H. (2010). The dissemination and implementation of evidence-bases psychological treatments. A review of currents efforts. American Psychologist, 65, 73-84.

Organización Mundial de la Salud (2005). WHO Multi-country-study on women's health and domestic violence against women summary report on prevalence, health outcomes and women responses. Ginebra: Organización Mundial de la Salud.

Pago, D. (2009). Comment: the evidence-based treatment debate: toward a dialectical rapprochement. Psychotherapy Theory, Research, Practice, Training, 46, 15-18.

Park, N., y Sun, J. K. (2013). La psicología positive: investigación y aplicaciones. Terapia Psicológica, 31, 11-19.

Rector, N. A., y Roger, D. (1993, julio). Self-concept and emotion-control. Presentado en el $3^{\text {rd }}$ Annual Meeting of the European Congress of Psychology. Helsinki, Finland.

Ruíz-Pérez, I., Plazaola-Castaño, J., Vives-Cases, C., Montero-Piñar, M. I., Escribà-Agüir, V., Jiménez-Gutiérrez, E., y Martín-Baena, D. (2010). Variabilidad geográfica de la violencia contra las mujeres en España. Gaceta Sanitaria, 24, 128-135.

Sanz, J., y Navarro, M. E. (2003). Propiedades psicométricas de una versión española del Inventario de Ansiedad de Beck (BAI) en estudiantes universitarios. Ansiedad y Estrés, 9, 59-84.

Sanz, J., Perdigón, A. L., y Vázquez, C. (2003). Adaptación española del Inventario para la depresión de Beck-II (BDI-II). 2. Propiedades psicométricas en población general. Clínica y Salud, 14, 249-280.

Sutherland, C. A., Bybee, D. I., y Sullivan, C. M. (2002). Beyond bruises and broken bones: The joint effects of stress and injuries on battered women's health. American Journal of Community Psychology, 30, 609-636.

Taft, A. J., y Hegarty, K. L. (2010). Intimate partner violence against women. What outcomes are meaningful? JAMA, 304, 577-579.

Tarragona, M. (2013). Psicología positiva y terapias constructivas: una propuesta integradora. Terapia Psicológica, 31, 115-125. 
Walker, L. (1994). Abused Women and Survivor Therapy. Washington, DC: APA.

Zlotnick, C., Capezza, N. M., y Parker, D. (2011). An interpersonally based intervention for low-income pregnant women with intimate partner violence: a pilot study. Archives of Womens Mental Health, 14, 55-65. 